Esta obra está bajo una Licencia Creative Commons Atribución-NoComercial-Compartirlgual 4.0 Internacional

(c) (i) (5) (2)

Apropiaciones y resistencias a la ESI en la Educación Física

Sofía Dueñas Díaz y Emilio José Tevez

DOI: $\underline{\text { https://doi.org/10.24215/16696581e273 }}$

Recibido: 09-02-2020 Aceptado: 13-03-2020

\title{
Apropiaciones y resistencias a la ESI en la Educación Física
}

\section{Appropriations and resistance to ESI in Physical Education}

\author{
Sofía Dueñas Díaz duediazsofia@gmail.com \\ https://orcid.org/0000-0002-4471-2873
}

Núcleo Regional de Estudios Socioculturales/ Facultad de Ciencias Sociales/ Universidad

Nacional del Centro de la Provincia de Buenos Aires, Argentina

\section{Emilio José Tevez tevezemilio@gmail.com \\ https://orcid.org/0000-0002-0491-7516}

Consejo Nacional de Investigaciones Científicas y Técnicas/ Núcleo Regional de Estudios Socioculturales/ Facultad de Ciencias Sociales/ Universidad Nacional del Centro de la Provincia de Buenos Aires, Argentina 


\section{Resumen}

Este artículo presenta algunas reflexiones sobre las complejidades que asume la implementación de la Ley de Educación Sexual Integral (ESI) en contextos cotidianos, atendiendo puntualmente a una iniciativa estatal que dispuso la forma mixta en la clase de Educación Física del nivel secundario. Se abordan las apropiaciones producidas por docentes y estudiantes en torno dicha modalidad mixta, focalizando el análisis sobre las resistencias expresadas por un grupo de estudiantes ante la modificación del contexto educativo. Su abordaje permitió reconocer que la oposición a la iniciativa estatal se debía a que la misma tensionaba la trama con la que se significaban las actividades desarrolladas en aquel contexto cotidiano. Acordando con los planteamientos propuestos por la ley, se busca analizar lo resistido intentando comprender aquello que se opondría a la ESI en espacios educativos. El trabajo se realizó desde un enfoque etnográfico a través de técnicas/estrategias como la observación participante y entrevistas, así como el análisis sobre fuentes documentales. El lugar de trabajo de campo corresponde con una escuela secundaria católica en una localidad de la provincia de Buenos Aires (Argentina), delimitando la población de estudio a docentes y estudiantes de $4^{\circ}$ a $6^{\circ}$ año.

Palabras Clave: género; educación; apropiación; resistencia.

\section{Abstract}

This article presents some thoughts on the complexities that the implementation of the Integral Sexual Education Law (ESI) assumes in everyday contexts, promptly attending to a state initiative that arranged the mixed- sex groups in Physical Education classes of the secondary level in the Buenos Aires province. Appropriations produced by teachers and students around this mixed modality are addressed, focusing on the analysis of the resistance expressed above all by a group of students in views of the modification of the educational context. Its approach allowed to recognize that the opposition to the state initiative was due to the fact that it tensioned the plot in which activities are developed and meant in the daily context. According to the stated by the law, we seek to analyze what is resisted, trying to understand what would oppose the ESI in educational spaces. The work was carried out from an ethnographic approach through techniques/strategies such as participant observation and interviews, as well 
as analysis of documentary sources. The field work takes place in a Catholic high school in a town of the of Buenos Aires province (Argentina), delimiting the study population to teachers and students from 4 th to 6 th year.

Keywords: gender; education; appropriation; resistance.

Introducción

Este trabajo propone reflexionar sobre las complejidades que asume la implementación de la ley Nacional № 26.150 de Educación Sexual Integral (en adelante ESI) en contextos cotidianos. El abordaje se focaliza en una iniciativa denominada clase mixta que estableció la integración de géneros en Educación Física, redefiniendo aquel espacio escolar que históricamente organizó el proceso educativo separando varones y mujeres. El objetivo es abordar las complejidades expresadas por los actores a partir de las apropiaciones que realizaron de la modalidad mixta, haciendo hincapié en las resistencias de un grupo de estudiantes ante la nueva política que plantea atender a la diversidad considerando la perspectiva de género. Lo que en principio pareciera ser aceptado sin mayores problemas, cambia cuando la propuesta se inmiscuye en la cotidianidad y desafía los significados que las personas atribuyen a ella. Principalmente, para quienes manifiestan la sensación de estar perdiendo algo que les daba sentido a las actividades desarrolladas en dicho contexto. Acordando con lo propuesto por la ley, se busca analizar lo resistido intentando comprender aquello que se opondría a la ESI en espacios educativos.

La investigación se ubica en el campo sub-disciplinar de la Antropología y Educación que, desde sus orígenes, brindó explicaciones alternativas a las posturas biologicistas/innatas otorgando un valor sustancial a la incidencia de la trama sociocultural en la constitución de las personas (Bernardi, 2000). Este principio se mantuvo a lo largo del tiempo considerando que «[...] todas las sociedades proveen algún tipo de entrenamiento y algún conjunto de criterios mediante los cuales los miembros pueden ser identificados como más o menos entendidos" (Levinson y Holland, 1996, s/n). Los entrenamientos y conjuntos de criterios se organizan bajo el concepto educación que refiere a un tipo de vínculo que ocurre cuando el agente interactúa con la trama de significados que organizan la vida cotidiana de un grupo. Las características que asume dicha interacción pueden ser comprendidas mediante la categoría apropiación que 
alude a cómo distintos actores se apropian de recursos culturales disponibles e interactúan en relación con la transformación de las fuerzas sociales que intervienen en el proceso, reinterpretando y transformando dichos recursos en el marco de las luchas sociales que caracterizan al contexto (Rockwell, 1996).

En estos términos, lo masculino y lo femenino no se define por aspectos innatos, sino a través de la trama sociocultural apropiada por las personas durante su experiencia cotidiana. Tal como lo muestra Margaret Mead (1973), características como el temperamento -atribuidas a las personas de acuerdo a su sexo- constituyen «[...] variaciones del temperamento humano, a las cuales pueden aproximarse por su educación, con más o menos éxito, según el individuo» ( $p$. 28). A catorce años de la sanción de la ESI esta, entre otras cuestiones, atraviesan la discusión en torno a su implementación y se presenta en la cotidianidad escolar bajo aspectos que, desde este trabajo, se consideran importantes para su análisis y reflexión. Principalmente, con aquellas voces que se resisten a esta política de ampliación de derechos considerando las posibilidades de extrañamiento y comprensión que brinda el enfoque socioantropológico. A continuación, el artículo se divide en cuatro apartados. El primero presenta el enfoque teórico y las categorías analíticas utilizadas. El segundo da cuenta del abordaje metodológico y las técnicas/estrategias de relevamiento, así como la descripción de la población de estudio y el contexto donde se realizó el trabajo de campo. En el tercer apartado, se desarrolla el análisis de las apropiaciones producidas sobre la modalidad mixta haciendo hincapié en las formas de resistencia. Por último, se presentan algunas reflexiones sobre el trabajo realizado.

\section{Aspectos conceptuales para abordar las apropiaciones/resistencias a la ESI}

Pablo Scharagrodsky (2004) analiza cómo el dispositivo escolar de Educación Física contribuyó desde su origen a la producción de ciertas masculinidades y feminidades mediante la distinción de prácticas corporales vinculadas con supuestos morales que definían a la feminidad a partir del pudor, la gracia y la delicadeza y a la masculinidad a partir de la virilidad, el coraje y la disciplina. Estos procesos de esencialización se apoyaron en razonamientos que confundieron atributos biológicos con condiciones morales y sociales. Desde esta confusión se sostuvo la subordinación y desvalorización de las mujeres y de varones que no cumplieran el guión masculino. Si bien actualmente no se distinguen prácticas corporales como se hacía anteriormente, aún persisten «[...] reglas invisibles y mecanismos ocultos que muy sutilmente van contribuyendo a construir cierto tipo de orden corporal y no otro» (p. 61) resultando que las diferencias jerarquizadas continúan instaladas en el ámbito escolar. 
Desde el campo de Antropología y Educación existen investigaciones que permiten complejizar esta cuestión, conceptualizando las apropiaciones y resistencias producidas en la implementación de la clase mixta. En primer lugar, estudios que abordan procesos de implementación afirman que los mismos no implican una imposición desde arriba, sino que se dan diferentes aspectos que «[...] entran en juego de manera no predecible y que le otorgan sentido local a las modalidades que finalmente asumirán los procesos de implementación de los programas» (Sinisi y Montesinos, 2009, p. 47). Esto supone reconocer que las iniciativas estatales son recibidas y reinterpretadas a partir de la apropiación que realizan las personas, la cual se caracteriza por aspectos que definen su carácter múltiple, relacional, transformativo y arraigado en conflictos sociales (Rockwell, 1996). Respecto a la categoría resistencia, la misma refiere a aquella fuerza que se opone a la que se considera la fuerza activa expresada en las acciones escolares y que en la escala cotidiana se puede manifestar, por ejemplo, en la indignación estudiantil ante lo pautado por el docente (Rockwell, 2006).

Un segundo nivel temático que permitió conceptualizar la cuestión refiere a estudios que han problematizado la perspectiva de género en clave educativa. Mead inauguró una de las principales corrientes en los estudios de género al analizar cómo se construye culturalmente lo femenino y lo masculino. Esta perspectiva fue complejizándose con aportes de distintos trabajos que pusieron en cuestión «[...] la matriz binaria masculino-femenino que configura la dualidad de los géneros en la cultura y en la historia» (Segato, 2010, p. 62). Así, se ampliaron los temas y comenzó a pensarse esta discusión en relación a una mayor diversidad de experiencias y configuraciones identitarias. La introducción del análisis sobre las construcciones de género en escuelas fue impulsada, entre otros, por estudios que mostraron cómo la presencia de sesgos diferenciadores -expuestos en los currículums explícitos, ocultos y evadidos- que perjudicaban a las mujeres (Morgade, 2007).

Bradley Levinson (1999) plantea que, si bien existe discriminación y los mensajes ideológicos aparecen como patrones normativos o estereotipos, estos también son comunicados a través de redes informales de la cultura estudiantil apoyada en ideologías de género que circulan dentro y fuera de la escuela. Una referente en la conceptualización del proceso en el que los actores se vinculan a los significados en torno a la masculinidad y feminidad en escuelas es Raewyn Connell (2001). Para la autora, la escuela constituye un agente institucional que participa del proceso donde se inscriben las estructuras y prácticas en las que se forman las masculinidades y un escenario en el que están en juego otros agenciamientos -especialmente el de los y las estudiantes-. Estos últimos «[...] participan en estas masculinidades con el simple hecho de entrar a la escuela y vivir en medio de sus estructuras. Sin embargo, los términos de 
esta participación son negociables -ya sea ajustándose a los patrones, rebelándose contra estos o tratando de modificarlos-» (p. 160).

Es importante tener presente que, así como conviven distintas masculinidades «[...] se refuerzan los sentidos de lo masculino definidos en su versión tradicional, soslayando o condenando la existencia de masculinidades subordinadas» (Morgade, 2007, p. 26). Así como la masculinidad hegemónica se impone ante aquellas subordinadas (Connell, 1997), lo mismo hace en los procesos de configuración de la feminidad. En estos términos, la masculinidad «[...]es al mismo tiempo la posición en las relaciones de género, las prácticas por las cuales los hombres y mujeres se compenetran con esa posición de género, y los efectos de esas prácticas en la experiencia corporal, en la personalidad y en la cultura» (Connell, 1997, s/n). Sobre ello se destaca cómo la diversidad (1) se construye en un marco de relaciones asimétricas a partir de una lógica binaria, atributiva y jerárquica presente en la cotidianidad escolar (Fernández, 2001). Con el objetivo de relevar la forma en que esta complejidad es expresada, se incorpora el concepto de procesos de alterización y desmarcación que Marcela Bilinkis y Mariana García Palacios (2013) utilizan para analizar los modos en que se producen, reproducen y resignifican las desigualdades de género. En este planteamiento, no existirían grupos que sean inherentemente otros, sino prácticas de alterización, de marcación de las diferencias y jerarquización de la diversidad, que sólo pueden comprenderse teniendo en cuenta la desigualdad social y los aspectos centrales de la estructura social (composición de clases, sistemas de producción y mercado de trabajo, distribución de poder, lucha ideológica y control cultural).

El enfoque teórico, proveniente del campo de Antropología y Educación, y las categorías apropiación y resistencia serán vinculados en el análisis con aspectos relevados durante el trabajo de campo referidos a las formas en que las personas reportan la realidad. Se analizarán entonces adaptaciones e invenciones creadas por el equipo docente para implementar la política así como reparos, oposiciones y cuestionamientos expresados por estudiantes a la forma mixta. El enfoque de género y las categorías referidas a lógicas y procesos mediante los cuales se producen y reproducen significados sobre la masculinidad y feminidad permitirán indagar sobre las ideas atribuidas al desempeño físico y temperamental de mujeres y varones en el deporte, así como su vínculo con lo que es: «serio/en joda» o «divertido/aburrido», cuestiones que incidieron en la forma en que las personas se apropiaron y resistieron a la política.

Question, Vol. 1, N. ${ }^{\circ}$ 65, abril 2020. ISSN 1669-6581

Instituto de Investigaciones en Comunicación | Facultad de Periodismo y Comunicación Social | Universidad Nacional de La Plata La Plata | Buenos Aires | Argentina

Página 6 de 17 


\section{Aspectos metodológicos, contexto y población de estudio}

El trabajo se realizó desde el enfoque etnográfico (Rockwell, 2009), siguiendo el principio de extrañamiento que establece la familiarización de lo exótico y la exotización de lo familiar (Lins Ribeiro, 2004). A partir de ello, se documentó lo no documentado permaneciendo en el campo a fin de registrar el proceso de implementación de las clases mixtas relevando el conocimiento local que los actores sociales producían. El protocolo de investigación constó de una serie de etapas: elaboración del proyecto (incluyó formulación de interrogantes, objetivos e hipótesis, lectura de antecedentes, diseño de estrategias de investigación), trabajo de campo (relevamiento de datos), análisis e interpretación de la información derivada del trabajo de campo y formulación de los resultados. Para la presentación de este artículo se han seleccionado algunos de los resultados producidos en la investigación.

El trabajo de campo se realizó entre marzo y noviembre de 2017 y se implementaron una serie de técnicas/estrategias de investigación (Achilli, 2005). En primer lugar la observación participante que «[...]consiste en dos actividades principales: observar sistemática y controladamente todo lo que acontece en torno al investigador y participar en una o varias actividades de la población» (Guber, 2001, p. 57). Esta estrategia es una herramienta para producir conocimiento logrado a partir de sus cruces con la teoría. Las observaciones fueron realizadas en la clase de Educación Física, permitiendo relevar características del contexto, formas de interacción, participación y experiencias de quienes participaban.

Respecto a la entrevista, Elena Achilli (2005) plantea que su objetivo es indagar sobre significados, perspectivas y definiciones de los actores. Es decir, sobre los modos en que las personas representan y experimentan la realidad. Inicialmente se realizaron entrevistas abiertas con diferentes actores de la escuela como la vicedirectora y tres docentes del área de Educación Física cuyas clases se observaron sistemáticamente. En las mismas se pudieron relevar apreciaciones y experiencias de los actores respecto a la redefinición de la clase, así como a sus temores y significados en torno al género. En los últimos dos meses se realizaron entrevistas con trece estudiantes, luego de lograr cierta confianza y establecer un criterio de selección para las mismas (a partir del cual se identificaron estudiantes que realizaban deporte fuera del espacio escolar, cuestión relevante para la investigación). La realización de las entrevistas estuvo condicionada -por autorización de la institución y familias- a hacerse en el mismo contexto de la clase. Ello posibilitó que durante las mismas los y las estudiantes vincularan los temas conversados con situaciones de la clase (ejemplificando escenas, señalando personas o situaciones, entre otras). Se realizaron con estudiantes entrevistas semiestructuradas, lo que orientó la indagación hacia temas particulares y posibilitó enfatizar sobre 
cuestiones relevantes para la investigación. Finalmente, se realizó un análisis de fuentes documentales, las cuales resultaron necesarias para comprender el contexto y las definiciones del proceso (Hammersley y Atkinson, 1994). Se indagó sobre documentos que el Estado nacional y provincial ha producido respecto al tema: leyes, resoluciones, diseños curriculares, informes del Ministerio de Educación; así como en documentos producidos por la propia escuela.

El ámbito donde se realizó el trabajo de campo correspondía a una escuela secundaria católica que llamaremos San Francisco (2). La implementación de la ESI comenzó a principios del año 2010 cuando un grupo conformado por el equipo directivo y algunos docentes de los distintos niveles elaboraron un documento denominado Programa de ESI (vigente durante la estancia de campo). El material fue enviado a los departamentos que coordinaban docentes de las distintas áreas donde se definieron las particularidades que asumiría la aplicación del programa dentro de sus correspondientes espacios curriculares. En este marco, durante el año 2017, comenzó a ser implementada la resolución № 2.476 del año 2013 emitida por la Dirección General de Cultura y Educación de la Provincia de Buenos Aires. La misma modificó la organización de las clases de Educación Física del nivel secundario, estableciendo la integración de géneros e incorporando la perspectiva de género y atención a la diversidad como aspectos a favorecer.

En la escuela San Francisco las clases de Educación Física tenían una hora de duración y se desarrollaban a contra-turno del horario escolar en un gimnasio ubicado en la parte posterior de la escuela. El lugar era amplio y estaba equipado para la práctica deportiva. En cada jornada, el gimnasio era compartido por dos y hasta tres grupos a la vez. Sobre esta multiplicidad de grupos, la población con la que se estableció interacción durante el trabajo de campo estuvo constituida por profesores, profesoras y estudiantes de $4^{\circ}$ a $6^{\circ}$ año. Los y las profesores Lucio, Camila y Flavio eran tres de los cuatro docentes que la escuela tenía para el área de Educación Física. Lucio (35 años), además trabajaba en otra escuela secundaria católicaprivada y en un gimnasio. Camila (32 años) trabajaba en el nivel secundario y primario de San Francisco. Flavio (42 años), además de profesor era entrenador de básquet en clubes de la ciudad. Los y las estudiantes que cursaban el $4^{\circ}, 5^{\circ}$ y $6^{\circ}$ año tenían entre 15 y 18 años. Cada grupo estaba conformado por entre 20 y 30 integrantes. La realización de observaciones sistemáticas así como las entrevistas permitieron acceder a la forma en que se identificaban y clasificaban entre sí, siendo una de aquellas diferenciaciones la que se establecía entre quienes no hacen nada o eran malas y malos en el deporte y quienes hacían o eran buenos deportistas. Sobre estos últimos se centró el interés, al ser las personas que hacían deporte fuera del espacio escolar (en clubes o Centros de Educación Física) quienes mayormente expresaron su oposición a la nueva forma.

Question, Vol. 1, N. ${ }^{\circ}$ 65, abril 2020. ISSN 1669-6581

Instituto de Investigaciones en Comunicación | Facultad de Periodismo y Comunicación Social | Universidad Nacional de La Plata La Plata | Buenos Aires | Argentina

Página 8 de 17 


\section{Apropiaciones y resistencias a la clase mixta}

La escuela San Francisco comenzó a implementar en 2010 su Programa de ESI. La vicedirectora explicó cómo fue el proceso afirmando que la cuestión se dificultó al tener que resolver asuntos que la reglamentación no explicaba: «lo que pasa es que hay cosas que la ley no dice. Hay muchas cosas que nosotros hemos tenido que inventar o aprender sobre la marcha». Uno de los casos a los que recurrió para ejemplificar lo inventado fueron las clases mixtas de Educación Física que comenzaron a realizarse por primera vez en 2016. Al inicio, en lugar de seguir la forma que establecía la resolución (conformar grupos de mujeres y varones por cada año), mantuvieron la organización de grupos divididos entre mujeres y varones de cada año y una vez cada tres meses realizaron un encuentro mixto. En los mismos, equipos conformados por mujeres y varones del mismo curso se enfrentaban en torneos. Según la vicedirectora, el objetivo fue comenzar de manera paulatina teniendo en cuenta que: «se habla del background de los alumnos pero después no se los escucha». Uno de los argumentos que justificaban esta escucha era la oposición estudiantil: «cuando hacíamos un encuentro empezaban con que los varones son brutos, que con las chicas es un embole». A pesar de estas resistencias, en el año 2017 y ante la exigencia de las autoridades educativas, comenzó la implementación tal como lo establecía la resolución conformando grupos mixtos por cada año en las clases semanales.

A partir de ello el departamento de Educación Física formuló su propio proyecto de ESI. Para fundamentarlo, se tomaron aspectos tanto del proyecto institucional así como de los lineamientos curriculares para el área -contenidos establecidos por el Ministerio de Educación de la Nación-. Según afirmó Camila el objetivo era: «lograr una igualdad, que aprendan a convivir... porque acá en la clase hay otros valores y otras normas de convivencia, porque al haber deporte y actividad física el contacto está y ahí se pone en juego el respeto». Para el equipo docente, los principales inconvenientes a la hora de reorganizar la clase fueron el contacto corporal entre mujeres y varones y la oposición por parte del estudiantado. Considerando esto, se realizaron modificaciones para adaptar la práctica con dos objetivos: regular el contacto entre varones y mujeres y lograr que jueguen todos. Esto modificó la organización de los deportes en la planificación anual (3) y, como comentó uno de los docentes, decidieron comenzar con vóley: «porque no es un juego de contacto, para ir de a poco... después vamos a seguir con handball que ahí va a ser distinto porque es de contacto y empiezan a decir yo con él no juego porque es bruto». A su vez, eligieron: «evitar el básquet porque cuando se marca hay más contacto y la práctica de fútbol: porque nos cuesta que las mujeres participen». Otra modificación fue la adaptación de los reglamentos deportivos: «en 
handball tiene que pasar la pelota por todos y el arquero cambia cuando le hacen un gol porque si no siempre se pasan la pelota entre los chicos o mandan a las mujeres al arco». Otra regla era que la pelota pique antes del gol para que valiera la anotación. Uno de los profesores agregó: «lo primero con lo que tuvimos que trabajar fue con el tema del bullying porque las chicas son más sensibles, si no las eligen cuando se arman los grupos se empiezan a sentir mal, que no sirven para el deporte».

De esta manera, la iniciativa de las clases integradas por género fue implementada a partir de la forma en que los y las docentes se apropiaron de ella. Mediante las adaptaciones que tuvieron que inventar porque no estaban explicitadas en la ley sortearon las dificultades $y$ temores. Al hacerlo transformaron la política, «[...]creando prácticas propias que no se asemejaba del todo al mandato oficial; más bien era una mezcla de su conocimiento de las costumbres y del lenguaje local, el sentido común pedagógico que habían heredado de sus mentores de la infancia» (Rockwell, 1996, p. 35). El equipo docente inventó las reglas con las que buscaron, además de evitar el contacto, suavizar la fuerza de los varones y otorgarles facilidades a las mujeres para que pudieran participar. Para esto, pusieron en juego sus propias interpretaciones sobre la diferencia de fuerza y actitud de los y las estudiantes que respondían a representaciones dominantes sobre el género. Con las reglas adaptadas se reproducían estereotipos en el marco de una lógica binaria, atributiva y jerárquica del género (Fernández, 2001) que estipulaba, por ejemplo, una habilidad natural en los varones para golpear la pelota con mayor fuerza. Así, se anticipaban comportamientos esperables para varones y mujeres, contribuyendo a la producción de procesos de marcación y alterización (Bilinkis y García Palacios, 2013).

Por su parte, las y los estudiantes tampoco recibieron pasivamente la iniciativa estatal sino que se apropiaron de la misma a partir de sus significados y prácticas. En el transcurso de las clases se dieron situaciones donde se expresaron reparos o cuestionamientos. Esto se observaba, por ejemplo, cuando debían agruparse para realizar ejercicios o jugar un partido. Los grupos, en general, eran conformados por un docente indicando que debían ser mixtos; cuando esta indicación no se realizaba, los grupos los armaban los mismos estudiantes agrupándose, en su mayoría, varones y mujeres separados. En una ocasión, por ejemplo, la profesora Camila indicó que se armaran grupos de cinco integrantes: «pónganse los varones de a dos y vayan tres mujeres a cada equipo". Los y las estudiantes se miraron y agruparon, algunos se llamaron expresamente, como un chico que llamó a una chica diciéndole: «Guillermina vení a jugar, necesitamos a Riquelme». Luego de la división, como la cantidad de varones y mujeres no era la misma -por ejemplo, quedaban cuatro varones con una mujer-, esta estudiante le pidió a la profesora: «¿no puede venir otra mujer?», la profesora hizo caso y 
llevó a una chica de otro grupo y mandó a un chico al otro. Luego de armar los grupos la profesora indicó: «un arquero y cuatro en cancha, yo voy a buscar la pelota y quiero que ustedes se organicen». Mientras se iban parando los grupos en la cancha un estudiante le gritó a otro que había quedado agrupado con cuatro mujeres: «jeh, maricón!», generando que el mismo abandone el grupo y se fuera a otro. Mediante estos comentarios, burlas o exigencias a la profesora, los y las estudiantes participaban en la conformación de grupos estableciendo pautas que no eran impuestas por docentes sino por ellas y ellos mismos. Así, un grupo de estudiantes podía convocar a jugar con ellos sólo a una chica que se destacaba jugando al fútbol afirmando que jugaba como un varón (Riquelme era jugador de fútbol del club Boca Juniors). Con burlas que apelaban a las representaciones sobre la masculinidad se nombraba maricón a aquel varón que no había sido identificado por la docente o jugara en un grupo solo con mujeres. Estos significados producidos en la organización de lo mixto los y las interpelaba, produciéndose reparo a participar del grupo que se indicaba integrar o solicitando que vaya otra mujer para no jugar en un grupo sola con los varones.

Tal como se relevó, la implementación del reglamento adaptado fue cuestionada por los y las estudiantes, lo cual se manifestó sobre todo en clases donde se enseñó handball y fútbol. Durante la explicación de las nuevas reglas se expresaron cuestionamientos que, en general, incluían la expresión: ¿por qué? de manera enfática. En un partido de handball un profesor explicó: «antes del gol, para que valga, la pelota tiene que pasar entre los cuatro jugadores. A la hora de lanzar al arco, lanzan con pique, no fuerte y directo». Un estudiante interrumpió y preguntó: «¿y si ataja un varón?» El profesor contestó: «igual, no importa, el arquero puede ser varón o mujer». Lo mismo en el partido de fútbol el profesor indicó: «arquero varón, el gol si es de las chicas vale tres». Una chica sonriendo dijo: «sí y si les pegamos no es falta». Cuando un chico escuchó esta última regla expresó su disconformidad: «iah profe! ¿por qué?» mostrándose enojado. Otro chico agregó -a modo de chiste-: «vamos a dársela siempre a las mujeres». El profesor no detuvo su explicación y siguió: «los laterales y los córner los hacen las chicas, y una chica dijo: «jah no!... ¿por qué?»

En el momento del juego los varones recibían la mayor cantidad de indicaciones sobre cómo proceder. Por ejemplo, a través de la insistencia docente: «jueguen con las nenas!» 0 : «ipasala! está Cande sola». Jugar con las nenas no fue el único señalamiento que se realizaba a los estudiantes. También se planteaba reiteradamente que jugaran suave con las chicas cuando, por ejemplo, un estudiante lanzaba la pelota al arco donde atajaba una mujer con mucha fuerza -según lo consideraba el docente-. Otro señalamiento se hacía cuando los estudiantes no respetaban la regla que establecía el pique en el piso de la pelota cuando se 
disparaba al arco. Docentes y algunos estudiantes lo expresaban a través de frases como: «eh, abusivo, bruto, jal arco con pique!, ¡suave!»

El grupo de estudiantes que realizaba deporte en espacios no escolares fue el que con mayor énfasis manifestó su resistencia. Frecuentemente, respondían con burlas cuando recibían la indicación de que jugaran suave o con las nenas. Así, cuando un docente les gritaba «isuave!», lanzaban la pelota exageradamente suave o expresaban: «pero si fue un pedito de vieja». También reían entre ellos y contestaban: «él es un varón pero con culo de mujer» para justificar, luego que el profesor les negara hacer un córner, por qué un chico iba a hacer algo que debían hacer las nenas. El humor, sin embargo, no era la única forma en que se expresaba la oposición. En diferentes ocasiones se observó cómo los estudiantes se mostraban visiblemente disgustados ante la intervención docente. Esto sucedió, por ejemplo, cuando un profesor exclamó: «juh!, igolazo!», luego que una jugadora lanzara la pelota al arco donde atajaba un varón cometiéndole un gol fuerte y sin pique. El hecho de que el docente no le llamara la atención a la jugadora por su infracción a la regla -y que incluso lo festejara-, fue reprochado por el arquero: «ah ¿no era de pique?», a lo que una de las jugadoras del equipo le contestó: «no pajero, si es mujer la que tira no». El profesor entonces, obligado a intervenir, expresó: «¿pero viste la fuerza que tiene? No, está bien, no valió», y le marcó el error a la estudiante: «icon pique!»

En estas situaciones la resistencia tomaba forma de una indignación que desafiaba abiertamente la aplicación de las reglas. Esto se vio, por ejemplo, en una ocasión durante un partido de handball. Primeramente, un estudiante protestó exclamando: «¿qué? ¡Yo no se la tiré fuerte!», luego de que uno de los profesores le gritara: «jey, suave!» al verlo tirar la pelota al arco donde atajaba una mujer. La indignación se expresó todavía más cuando, minutos después de esa jugada, el mismo estudiante ocupando la posición de arquero recibió el lanzamiento sin pique de una chica marcándole gol. Ante un nuevo silencio docente que no sancionó a esta jugadora, el estudiante reclamó: «jey! ¿Vio? ¿Ellas sí pueden tirar fuerte?»De la misma manera sucedió en los partidos de fútbol cuando se aplicó la regla de que el gol de las mujeres valía doble. Al término de un partido donde una chica había metido dos goles y un chico del equipo contrario uno, cuando el profesor comunicó que el resultado de acuerdo al reglamento adaptado era 6 a 1, uno de los estudiantes del equipo perdedor se quejó: «jah no! no vale, metieron dos goles y fueron seis". La presencia de mujeres que metían goles o tiraban con fuerza, a la vez que contradecía los estereotipos que justificaba la adaptación del reglamento, significaba para los estudiantes una demostración de lo injusto de las nuevas reglas. La burla y la indignación eran maneras en que los estudiantes resistían a la nueva 
forma, relacionando y confrontando «[...] lo dicho por el maestro con su propia experiencia y pensamiento, lo cual puede llevar a cuestionar lo que se presenta» (Rockwell, 2006, p.33).

En las entrevistas, este grupo de estudiantes argumentó las razones de su oposición a la nueva política, pudiéndose apreciar cómo las representaciones de género influyeron sobre la forma en que los mismos la recibieron. En este sentido, pese a la heterogeneidad de los grupos en los que había mujeres que participaban activamente de los partidos y varones que no se esforzaban demasiado en el juego (4), la pasividad de no moverse era asociada a los comportamientos de las mujeres. Este punto fue fundamental para este grupo que consideraba que la energía y el esfuerzo en la práctica deportiva era importante y se lo exigían a quienes, según su criterio, no lo demostraban en los partidos. Un estudiante de $6^{\circ}$ año fundamentó este posicionamiento:

Nosotros veníamos desde primero haciendo los varones siempre juntos y es otra cosa. Venimos, transpiramos como locos, corremos, hacemos de todo, y después al unirte con las chicas y que no se muevan ni nada como que te chocaba. Ahora ya es un cago de risa, nos acostumbramos a que por ahí ellas no se la toman como una clase posta, vienen para cumplir, para no tener la falta, por no llevarse la materia.

Uno de los aspectos que destacaban refería a tener que jugar más suave, lo que significaba verse impedidos de jugar como lo hacían en otros contextos. Dentro de este grupo se entendían los motivos de los cambios:

\begin{abstract}
Ahora capaz que se hace un poco más tranquilo porque los juegos son un poco menos brutos porque como que los varones a veces se exceden de fuerza y las chicas...no todas, porque hay mujeres que juegan así como los varones, no es de machista ni nada pero a veces a las mujeres no podés ir a chocarlas con la misma fuerza que a un varón jugando al básquet, al fútbol o al handball. Jugar a divertirte sería, no siempre a intentar superar al rival.
\end{abstract}

Las reglas adaptadas establecían que los varones debían limitar su fuerza lo que implicaba cambiar el significado que comúnmente le otorgaban a la práctica deportiva. Esto iba en contra de los intereses de este grupo porque, como planteó otro estudiante de $5^{\circ}$ año:

\begin{abstract}
A los varones nos gusta jugar agresivo, jugar más al contacto, al pegar un poco más... con las mujeres tenés que jugar más despacio, tenés que sacar de abajo, tenés que... entonces es como que se vuelve aburrido, estás muy quieto... ponele, jugamos al handball y estamos ahí muy parados, no hacés nada. En cambio cuando jugamos entre varones vas así, lo empujaste, le pegás un codazo y así tiras con todo...te importa menos digamos que si hay una mujer en el arco por ahí tenés que tirar más despacio por si le pegás.
\end{abstract}


Estos estudiantes preferían la forma anterior y para argumentar su oposición se valían de representaciones de género dominantes al contraponer el juego entre varones del juego con mujeres: la clase con mujeres se volvía un cago de risa porque no se lo tomaban como una clase posta, en oposición a cómo estos varones entendían su propia práctica. Lo divertido del juego mixto resultaba de quitar el sentido del deporte competitivo vinculado a intentar superar al rival. Sin contradecir esta definición, a la vez se sostenía que el juego mixto se volvía aburrido porque con las mujeres tenían que estar quietos y jugar despacio, en oposición a la forma agresiva del juego entre varones. Reivindicando lo divertido/serio del juego entre varones y oponiéndolo al juego aburrido/cago de risa con las mujeres, justificaban su rechazo a la nueva política.

El equipo docente sabía que este grupo era el que había desaprobado desde un primer momento las clases mixtas. Según un profesor: «Tenían la idea de jugar siempre en forma más intensa o de hacer algún deporte que ahora no lo podés hacer o si lo hacés, lo hacés más reducido o diferente». Transcurrido un tiempo, el mismo docente destacó que: «aunque no les guste, se dieron cuenta que los deportes los podíamos hacer igual y que muchas veces toda la competitividad que tenían cuando lo hacían descendía pero igual lo pasaban bien». Como se puede apreciar en las afirmaciones de este docente, la resistencia era producto de la sensación de haber perdido algo con la nueva organización de la clase: la forma intensa y la competitividad al jugar. En este sentido, los estudiantes continuaban sosteniendo que la clase mixta los perjudicaba y era un límite para su desarrollo deportivo; así lo expresaba un estudiante de $6^{\circ}$ año:

\begin{abstract}
A las mujeres las benefician porque juegan con nosotros que somos más agresivos y eso en el deporte está bueno. Pero nosotros cuando jugamos al voley queremos rematar fuerte y por ahí hay una chica que no sabe defenderse y como que te abstenés de eso. Entonces te perjudica a vos porque no mejorás... no te esforzás porque tenés límite, a veces te marcan ese límite. A mí no me gusta que me pongan un límite.
\end{abstract}

Al analizar la resistencia se destaca cómo, para el grupo de varones deportistas, la política de integración de géneros implementada por el equipo docente a través de las reglas adaptadas con eje central en desarrollar la práctica del deporte más suave- fue considerada negativa. En las entrevistas los estudiantes daban cuenta de aquello que perdían con la forma mixta; el énfasis de la oposición respondía a que se quitaba la seriedad y exigencia que había caracterizado a la clase previamente cuando era divertida, es decir, solo de varones. Las reglas, aunque continuaban reproduciendo estereotipos de género, entraban en tensión con los sentidos asociadas a la forma masculina de jugar. 


\section{Consideraciones Finales}

En este trabajo se analizó la complejidad que asumió la implementación de la resolución que dispuso la integración de géneros en la clase de Educación Física de una escuela secundaria. Recuperando lo planteado por Sinisi y Montesinos (2009), el proceso de implementación estuvo atravesado por aspectos que le otorgaron un sentido local. Aquí se abordó particularmente la forma en que dicha política fue recibida y reinterpretada por docentes y estudiantes en el marco de su experiencia cotidiana, cuestión que expresó diferentes aspectos vinculados al carácter multidimensional del proceso de apropiación (Rockwell, 2005). En el caso estudiado, se identificaron tensiones entre lo que proponía la resolución y cómo las personas la experimentaron en relación a las formas de significar lo femenino y lo masculino, lo cual condicionó el proceso y transformó aquello que se imponía con la política. Para el equipo docente, esto significó un desafío ya que había cuestiones que la ley no explicitaba cómo se debían resolver, lo que generaba temores que llevaron a adaptar distintos aspectos de la clase. Con sus propias reglas, estos se apropiaron activamente de la propuesta, reinterpretando y transformando su contenido original. Así, la adaptación de reglas realizada por docentes, pese a desarrollarse en el marco de un proyecto que proponía formas de igualdad, reproducía significados asociados a una lógica jerárquica del género.

Tal como se planteó, los y las estudiantes no recibieron pasivamente la propuesta sino que participaron activamente del proceso poniendo en juego sus propias pautas y criterios. Aquí se hizo hincapié en las resistencias de un grupo de estudiantes quienes, a través de burlas y expresiones de indignación, manifestaban su desacuerdo con la forma mixta. La resistencia se debía a que jugar con mujeres iba en contra de la trama de significados que organizaba y daba sentido al contexto cotidiano. La fuerza de esta trama radicaba en que definía lo que para ellos resultaba serio y divertido. Los estudiantes tenían un modo de significar las actividades que desarrollaban en aquel contexto lo que hacía que la propuesta de la ESI se valorara como algo poco serio y aburrido.

El abordaje conceptual realizado permitió vincular en el análisis aquello conceptualizado en términos de apropiaciones y resistencias con la trama de significados en torno al género presente en lo cotidiano de la clase. Esto resulta importante considerando que aquellos significados que históricamente justificaron la separación de varones y mujeres en la clase continuaban estando presentes pese a que se hubiera cambiado su organización. Mediante este análisis es posible construir nuevos interrogantes para indagar en aquello que se opone a la ESI en el espacio educativo, teniendo presente que la cuestión de género puede estar implícita en las formas que los actores tienen de entender lo que es serio o divertido. Esto 
último permitiría pensar iniciativas en el marco de la ESI que busquen trabajar con quienes se resisten a la política e interpelar lógicas binarias y jerárquicas del género en aquellas instancias donde pueden aparecer más opacadas.

Notas

(1) El concepto de diversidad «[...]ha sido utilizado de modos diferentes, apelando a distintos sentidos; en muchas ocasiones, inclusive, como una manera de encubrir las relaciones sociales desiguales» (Bilinkis y García Palacios, 2013, s/n).

(2) Los nombres de la institución así como de las personas involucradas han sido modificados para preservar su identidad.

(3) La planificación anual era un documento producido por docentes donde se establecían contenidos -establecidos en el diseño curricular elaborado por el Ministerio de Educación de la Provincia- a desarrollar en el marco de una secuencia didáctica.

(4) Si bien se considera esta heterogeneidad de experiencias, a los fines del tema del artículo así como su extensión se destaca en el análisis lo que sucedía con los varones que realizaban deporte fuera de la escuela, al ser aquellos que con más énfasis se resistieron a la forma mixta.

\section{Referencias bibliográficas}

Achilli, E. (2005). Investigar en Antropología Social. Los desafíos de transmitir un oficio. Rosario: Laborde.

Bernardi, G. (2000). De las sociedades exóticas a la "exotización" del aula: una aproximación a la problemática educativa dentro de la antropología. Ponencia presentada en las IV Jornadas Rosarinas de Antropología Social de la Universidad Nacional de Rosario. Rosario, Argentina. Recuperado de https://www.equiponaya.com.ar/congresos/contenido/4rosario/jornadas/ANTROPOLOG IA\%20Y\%20EDUACION/antropologia\%20y\%20educacion\%20Bernardi.pdf

Bilinkis, M. y García Palacios, M. (2013). Juego, niñez y género en la escolarización inicial. Reflexiones a partir de la capacitación docente. Lúdicamente, 2 (4). Recuperado de: http://biblioteca.clacso.edu.ar/Argentina/iigg-uba/20140708045051/3116-17241-1PB.pdf

Connell, R.W (1997). La organización social de la masculinidad. En Valdés y Olavarría (editores), Masculinidad. Poder y crisis. Santiago de Chile: Isis Internacional. 
Connell, R.W. (2001). Educando a los muchachos: nuevas investigaciones sobre masculinidad y estrategias de género para las escuelas. Nómadas, 14, 156-171.

Fernández, Ana M. (2001). El fin de los géneros sexuales. S/d.

Hammersley, M. y Atkinson, P. (1994). Etnografía. Métodos de investigación. Barcelona: Paidós.

Lins Ribeiro, G. (2004). Descotidianizar. Extrañamiento y conciencia práctica, un ensayo sobre la perspectiva antropológica. En: Arribas V., Boivin M. y Rosato, A (edit), Constructores de otredad. Una introducción a la Antropología Social y Cultural (pp. 194-198). Ciudad de Buenos Aires: Antropofagia.

Levinson, B (1999). Ideologías de género en una escuela secundaria mexicana: hacia una práctica institucional de equidad. Revista Latinoamericana de Estudios Educativos, 2 (29).

Levinson B. y Holland D. (1996). The Cultural Production of the Educated Person: An Introduction. En: Levinson B.; Foley D. y Holland D. (eds.)The cultural production of the educated person. Critical Ethnographies of Schooling and Local Practice. New York: University of New York press.

Mead, M. (1973). Sexo y temperamento en las sociedades primitivas. Barcelona: Lata.

Montesinos, M. y Sinisi, L. (2009). Entre la exclusión y el rescate. Un estudio antropológico en torno a la implementación de programas socioeducativos. Cuadernos de Antropología Social, 29, 43-60.

Morgade, G. (2007). Educación, relaciones de género y sexualidad: caminos recorridos, nudos resistentes. Género, generación y etnicidades en los mapas educativos contemporáneos. Subsecretaría de Educación de la DGCyE.

Rockwell, E. (1996). Claves para la apropiación: la educación rural en México. En: Levinson B.; Foley D. y Holland D. (eds.)The cultural production of the educated person. Critical Ethnographies of Schooling and Local Practice. New York: University of New York press.

(2006). "Resistencia en el aula: entre el fracaso y la indignación". Educaçao em revista, 44, 1339.

(2009). La experiencia etnográfica: historia y cultura en los procesos educativos. Buenos Aires: Paidós.

Scharagrodsky, P. (2004). Juntos pero no revueltos. Cadernos de Pesquisa, 34 (121), 59-76.

Segato, R. (2010). Las estructuras elementales de la violencia. Ensayos sobre género entre la antropología, el psicoanálisis y los derechos humanos. Buenos Aires: Prometeo. 